\title{
О СТАТИЧНОЙ/ДИНАМИЧНОЙ ВАРИАНТНОСТИ СЕМЕМ АНГЛИЙСКИХ ПРОСТРАНСТВЕННЫХ НАРЕЧИЙ
}

\author{
М. Е. Панкратова \\ Воронежский институт Министерства внутренних дел Российской Федерации
}

\author{
ON STATIC/DYNAMIC VARIANTS OF SEMEMES \\ OF ENGLISH SPATIAL ADVERBS
}

\author{
M. E. Pankratova \\ Voronezh Institute of the Ministry of Internal Affairs of the Russian Federation
}

\begin{abstract}
Аннотация: статья посвящена изучению семантики английских пространственных наречий, имеющих статичные и динамичные варианты употребления. Изучение семантики наречных слов представляет собой большую трудность из-за специфики данной части речи в английском языке. В исследовании рассматриваются нюансы работы с данным классом слов, анализируются существующие классификации английских пространственных наречий и подходы к разграничению пространственных наречий на наречия места и перемещения. Делается предположение о невозможности построения однозначной семантической классификации ввиду превалирования функиионально-позиционного критерия разграничения частей речи в английском языке над семантическим. Способность английского наречия описывать статичные и динамичные ситуачии понимается как семантическая вариантность. Предлагается классификация пространственных наречий на основе их реального функционирования в предложении и способности одного и того же наречия обозначать ситуачии местонахождения и перемещения в зависимости от сочетания с глаголом. Материалом исследования послужили 96 пространственньх наречий, зафиксированных в словарях. Методами семного и семемного анализа были выделены пространственные семемы, среди которых были отмечены значения, способные употребляться в статичных и динамичных вариантах. Реальное функиионирование данных семем в речи было изучено в контекстах, взятых из Британского национального корпуса. С помощью сопоставительно-параметрического метода лингвстических исследований и предлагаемых в его рамках индексов и шкал была проанализирована семантика означенных наречий и сделаны выводы об ее особенностях, предложены семантические типы наречий с учетом превалирования статичных или динамичных вариантов значений.
\end{abstract}

Ключевые слова: английские пространственные значения, сопоставительно-параметрический метод, статичные и динамичные варианты значений, индекс, пространственная семема.

\begin{abstract}
English spatial adverbs that have static and dynamic variants. The study of the semantics of adverbs is a complicated process in the English language. The paper examines the peculiarities of this class of words, analyzes the existing classifications of English spatial adverbs and approaches to categorization of spatial adverbs into adverbs of place and movement. An assumption is made about the impossibility of constructing an unambiguous semantic classification due to the prevalence of the functional-positional criterion for differentiating parts of speech in English over the semantic one. The ability of English spatial adverbs to describe static and dynamic situations is understood as semantic variation. A classification of spatial adverbs is proposed on the basis of their real functioning in a sentence and the ability of the same adverb to designate situations of location and movement, depending on a verb-adverb combination. 96 spatial adverbs recorded in dictionaries have been used as the material of this study. The methods of seme and sememe analysis have been used to identify spatial sememes with static and dynamic variants. The actual functioning of these sememes in speech has been studied in contexts taken from the British National Corps. Using the comparative-parametric method of linguistic research and the indices and scales proposed within this method, the semantics of the adverbs has been analyzed and conclusions about its peculiarities have been drawn, semantic types of adverbs have been proposed.
\end{abstract}

Key words: English spatial adverbs, comparative-parametric method, static and dynamic variants of sememes, index, spatial sememe.

(C) Панкратова М. Е., 2021

Контент доступен под лицензией Creative Commons Attribution 4.0 License.

The content is available under Creative Commons Attribution 4.0 License. 


\section{Введение}

Широко известен тот факт, что одно и то же английское слово может быть причислено к различным частеречным категориям в зависимости не столько от семантики лексемы, сколько от ее позиции и функции в предложении [1;2]. С этой точки зрения, такая часть речи, как английское наречие, является наиболее сложным для изучения классом слов, поскольку его высокая гибридность, текучесть границ и тесная связь с местоимениями, прилагательными, частицами и предлогами усложняет любые исследования в данной области, особенно - в области их значений [3]. Так, существует огромное число семантических классификаций наречий, в которых ученые выделяют от 3 до 11 типов наречных слов (подробнее об этом см.: [4]). Примечателен и вполне логичен тот факт, что пространственные наречия (в другой терминологии «локативные», или наречия места) выделяются во всех классификациях, поскольку они обозначают базовые онтологические понятия ориентации человека в пространстве [5, с. 30-33].

Однако при дальнейшей классификации пространственных наречий языковеды сталкиваются с определенными трудностями. Некоторые лингвисты подразделяют английские локативные наречия на наречия места/расположения, направления/движения/ перемещения [1; 6]. Другие - не выделяют среди наречий места подтипы наречий местонахождения и перемещения, утверждая, что при учете особенностей функционально обусловленной отнесенности английских слов к той или иной части речи одна и та же лексема может попадать как в разряд наречий расположения, так и движения (to be upstairs, to go upstairs) $[2 ; 7]$.

В отечественной англистике такая поликатегориальность наречных лексем изучается в рамках сопоставительно-параметрического метода лингвистических исследований, развиваемого в Воронежском государственном университете [4; 8; 9]. Способность наречия обозначать местоположение или движение предмета в зависимости от сочетания с глаголом движения или местонахождения понимается в рамках данного подхода как статичность/ динамичность пространственного значения [8, с. 27]. Таким образом, поликатегориальный подход рассматривает обозначение местонахождения и перемещения как вариантность одного и того же пространственного значения. В данной статье мы рассмотрим феномен полифункциональности английских пространственных наречий, способных обозначать как местонахождение предмета в пространстве, так и его перемещение, и сделать выводы об особенностях их семантики.

\section{Материалы и методы исследования}

Материалом исследования послужили 96 наиболее частотных пространственных наречий современного английского языка, зафиксированные в толковых словарях и словарях частотности [10; 11-13]. Это наречия above, aboard, abroad, across, afar, afield, aground, ahead, aloft, along, alongside, anywhere, around, aside, ashore, astern, away, back, backstage, backwards, below, behind, beyond, centrally, close, deep, down, downhill, downstairs, downstream, downwards, eastwards, elsewhere, everywhere, far, fore, forwards, halfway, here, high, home, in, inboard, indoors, inland, inside, inshore, internally, inwards, inwardly, leewards, low, near, nigh, north, north-eastwards, northwards, nowhere, on, out, outside, outwards, overland, overseas, off, over, overboard, overhead, posteriorly, remotely, right, round, seawards, shorewards, somewhere, south, southwards, south-eastwards, there, thereabouts, therein, under, underfoot, underground, underneath, uphill, upstairs, upstream, upwards, west, westwards, where, wherever, windwards, within, yonder.

На первоначальном этапе исследования методом анализа словарных дефиниций, а также методами семного и семемного анализа нами были выделены все пространственные семемы изучаемых наречий (т. е. семемы, отвечающие на вопросы «где?», «откуда?», «куда?» [8, с. 12]). Таких значений оказалось 373.

Далее с помощью метода корпусного анализа нами были изучены 1000 контекстов употребления каждой наречной лексемы в Британском национальном корпусе [14], подсчитано количество употреблений пространственных семем, а среди них выделены статичные (отвечающие на вопрос «где?») и динамичные (отвечающие на вопросы «куда?», «откуда?») варианты употребления каждой пространственной семемы.

Введенный в рамках сопоставительно-параметрического метода лингвистических исследований индекс статичности/динамичности семемы, под которым понимается отношение количества зафиксированных статичных/динамичных употреблений семемы к общему количеству ее зафиксированных употреблений [4, с. 39], позволяет сделать выводы о том, какой вариант семемы - статичный или динамичный - является более часто встречающимся и, следовательно, более коммуникативно востребованным на современном этапе развития английского языка.

Интерпретировать значения индексов помогает разработанная Л. А. Кривенко «Шкала ранжирования степени выраженности исследуемых параметров» [15]. Согласно данной шкале, если показатель индекса равен нулю, т. е. рассматриваемое явление отсут- 
ствует, мы говорим о нулевой степени проявления признака. Если показатели имеют значения в пределах от 0,1 до 1, степень выраженности исследуемого явления признается сверхнизкой, от 1 до 10 - низкой, от 10 до 30 - заметной, от 30 до 50 - яркой, от 50 до 70 - значительной, от 70 до 90 - высокой, от 90 до 100 - гипервысокой. Если же индекс имеет значение 100 \%, это свидетельствует об абсолютной степени выраженности исследуемого параметра.

На завершающем этапе исследования нами была введена шкала ранжирования степени статичности/ динамичности пространственных семем, которая позволяет интерпретировать показатели степени проявления статичности и динамичности семемы и сделать выводы о типе рассматриваемого пространственного значения в зависимости от его способности в большей степени описывать местоположение или движение (таблица).

Так, семема является в равной степени статичной и динамичной, если показатели индексов статичности и динамичности являются яркими и равны $50 \%$. Семему стоит признать в большей степени статичной или динамичной при показателях соответствующего индекса больше 50 \%. Абсолютная степень динамичности или статичности характеризует семему как исключительно динамичную или статичную.

Т а б ли ц а

Шкала ранжирования степени статичности/динамичности пространственных семем

\begin{tabular}{|c|c|c|}
\hline $\begin{array}{c}\text { Численные значения индексов } \\
\text { статичности/динамичности }\end{array}$ & $\begin{array}{c}\text { Степень выраженности } \\
\text { статичности/динамичности }\end{array}$ & Тип семемы \\
\hline $0 / 100 \%$ & Нулевая / абсолютная & Исключительно динамичная \\
\hline $\begin{array}{c}>050 \% / \\
>51 \%<100 \%\end{array}$ & $\begin{array}{c}\text { Сверхнизкая, низкая, заметная, яркая / } \\
\text { значительная, высокая, гипервысокая }\end{array}$ & В большей степени динамичная \\
\hline $50 \% / 50 \%$ & Значительная & $\begin{array}{c}\text { В равной степени статичная } \\
\text { и динамичная }\end{array}$ \\
\hline $\begin{array}{c}51 \%<100 \% / \\
>0<50 \%\end{array}$ & $\begin{array}{c}\text { Значительная, высокая, гипервысокая / } \\
\text { сверхнизкая, низкая, заметная, яркая }\end{array}$ & В большей степени статичная \\
\hline $100 \% / 0$ & Абсолютная / нулевая & Исключительно статичная \\
\hline
\end{tabular}

\section{Обсуждение результатов}

Рассмотрим процедуру определения степени статичности/динамичности семемы на примере наречий below, above, elsewhere, underfoot, которые позволяют продемонстрировать все возможности применения сопоставительно-параметрического метода для изучения лексем: определения особенностей семантики и функционирования семем, выявления отмирающих и появляющихся новых значений.

Лексема below, согласно данным словарей, насчитывает семь пространственных семем: «внизу/вниз» (to be placed below / to go below), «в нижнем этаже» (a bedroom below), «ниже по течению» (there's good fishing below), «в нижней части» (blue on top and green below), «в аду» (he's not in heaven, he's below), «ниже в тексте» (see below), «на более низкой ступени социальной лестницы» (major and below). Как видно, среди перечисленных значений словари фиксируют только одну семему, обладающую статичным и динамичным вариантом употребления: «внизу/вниз».

Обратившись к данным корпусной лингвистики и проанализировав примеры употребления лексемы below, мы обнаружили следующее. У семемы «внизу/ вниз» наиболее востребованным оказался ее статичный вариант: From up here you can see the cows browsing in the fields below. Индекс статичности имеет показатель 93,6 \%, степень статичности семемы является, таким образом, гипервысокой. На долю динамичного варианта приходится 6,4 \% употреблений семемы, степень динамичности можно охарактеризовать как низкую: Don't look below. Шкала ранжирования степени статичности/динамичности семемы позволяет отнести данную семему к типу в большей степени статичных.

Анализ контекстов из Британского национального корпуса позволил установить, что семема «в нижнем этаже» (on the floor below), зафиксированная в словарях исключительно как статичная, имеет на самом деле и динамичный вариант употребления: The nurse had gone below for a meal. При этом динамичный вариант оказался коммуникативно высоко востребованным, индекс динамичности семемы равен 90 \%, тогда как статичный вариант встречается только в $10 \%$ случаев, что говорит о том, что данная семема является в большей степени динамичной.

Как видно из рассмотренных примеров, лексема может развивать несколько пространственных значений, одно из которых может быть, согласно шкале ранжирования степени статичности/динамичности, в большей степени статичным, а другое - динамичным, поэтому относить изолированное наречие к какой-либо конкретной семантической группе не 
представляется правильным. Кроме того, анализ контекстов из Британского национального корпуса позволил выделить не зафиксированные в словарях варианты значений.

Проведенное исследование показало, что у некоторых наречий превалирующей оказалась статическая семантика. Рассмотрим это на примере лексемы above.

Наречие above имеет 6 пространственных значений: «наверху/наверх» (to stay above / we were led above), «на стороне спины (зоол.)» (this lizard is green above), «выше в тексте» (read above), «на более высокой ступени карьеры» (the rank of Sergeant and above), «на небе / на небо» (eternal rest above / he has gone above), «выше по течению» (there's good fishing above). Как видно из словарных примеров, статичную/ динамичную вариантность развивают две пространственные семемы: «наверху/наверх», «на небе / на небо». Однако данные корпусной лингвистики свидетельствуют об иных результатах. Семема «наверху/ наверх» встретилась нам исключительно в статичном варианте «наверху» (Ahead and above I could see the famous Bissolotti castle). Индекс статичности, таким образом, равен $100 \%$, степень проявления рассматриваемого признака стоит признать абсолютной, а семему - исключительно статичной. Аналогичным образом, динамичный вариант значения «на небо» в выборке не встретился и может быть признан коммуникативно нерелевантным, свидетельствуя об абсолютной степени статичности рассматриваемой семемы (Do you have faith in God above?).

Таким образом, анализ контекстов позволяет выявить реально востребованные на современном этапе развития английского языка варианты пространственных значений, свидетельствуя не только о появлении новых вариантов семем, но и об отмирании уже известных семем.

Превалирование динамичных вариантов значений рассмотрим на примере наречия elsewhere.

Лексема elsewhere обладает одной пространственной семемой «в другом месте / в другое место» (to be elsewhere / to go elsewhere), демонстрируя наличие как статичного, так и динамичного вариантов употребления.

Анализ контекстов показал, что превалирующим является динамичный вариант семемы (You'll have to go elsewhere for a better life), индекс динамичности имеет гипервысокий показатель 91,8 \%, тогда как индекс статичности является низким и составляет 8,2 \% (I have to live elsewhere). Согласно шкале ранжирования степени статичности/динамичности семемы, данное значение является в большей степени динамичным.

Интересным с точки зрения результатов анализа является наречие underfoot. Данная лексема имеет две пространственные семемы, обе семемы имеют статичный и динамичный варианты: «под ногами / под ноги» (to be underfoot / to put underfoot) и «под кораблем / под корабль (мор.)» (water underfoot / to throw underfoot).

Согласно данным Британского национального корпуса, статичный вариант семемы «под ногами» (It was slushy underfoot) характеризуется индексом статичности, равным 97,6 \%. Степень выраженности данного признака является гипервысокой, следовательно, данное значение стоит признать в большей степени статичным. Индекс динамичности составил 2,4 \%, степень проявления означенного признака стоит признать низкой (He threw the beads underfoot).

Что касается семемы «под кораблем / под корабль (мор.)», то она оказалась в равной степени статичной и динамичной, оба индекса имеют значительный показатель 50 \%: There was a strong current underfoot (статичный вариант) / The ship was surrounded by smaller vessels throwing mackerel underfoot (динамичный вариант).

В целом исследование показало, что из 373 пространственных значений 131 семема $(35,1 \%)$ обладает статичными и динамичными вариантами употребления. При этом динамичные варианты употребления оказались превалирующими: из 131 семемы у 74 (56,5 \%) более востребованными оказались динамичные варианты употребления, тогда как статичные - у 55 семем (41,9 \%). Лишь одна семема оказалась в равной степени статичной и динамичной $(0,8 \%$ от общего числа семем), и одна - исключительно статичной $(0,8 \%)$.

В ходе проведения исследования было выявлено шесть динамичных и три статичных варианта семем, не зафиксированных в словарях. Помимо описанного выше значения лексемы below, это динамичные варианты семем «на другой конец, в другую сторону» лексемы over (She went over to fetch a cup of coffee); «вниз» лексемы underneath (Jasper ducked underneath), «до середины отрезка» лексемы halfway (Dip in a rooting aid and then poke halfway in), «в центр» лексемы centrally (Position the mistletoe centrally), «домой» лексемы in (She kissed me when I came in from work).

К появляющимся новым статичным вариантам значений отнесем семему «в прежнем состоянии» наречия back (I am back to my usual self), «в месте повышенной значимости» лексемы over (Over in Nottinghamshire, they are marking their elevation to the Central Midlands League), «в учреждении» лексемы in (No one is ever in before noon).

Превалирование динамичной семантики среди традиционно выделяемых словарями значений, а также среди возникающих новых семем можно объяснить общим стремлением языка к развитию. 


\section{Заключение}

В целом исследование английских пространственных наречий, обладающих статичными и динамичными вариантами значений, с применением сопоставительно-параметрического метода позволило выявить особенности их семантики и функционирования, определить тип каждой из семем и классифицировать их как в большей степени статичные или динамичные, в равной степени статичные и динамичные, исключительно статичные. Исключительно динамичных семем нами выявлено не было.

Результаты исследования доказывают невозможность однозначной классификации наречий по семантическим типам ввиду многозначности слов и полифункциональности английских пространственных наречий. Представляется целесообразным рассматривать не лексему, а ее отдельно взятые семемы, статичную и динамичную вариантность.

\section{ЛИТЕРАТУРА}

1. Quirk R. et al. A Comprehensive Grammar of the English Language. L. : Longman, 1985. 1779 p.

2. Carter R., McCarthy M. Cambridge Grammar of English. A Comprehensive Guide to Spoken and Written English Grammar and Usage. Cambridge : Cambridge University Press, 2007. 973 p.

3. Хаймович Б. С., Роговская Б. И. Теоретическая грамматика английского языка (на английском языке). М. : Высшая школа, 1967. 298 с.

4. Панкратова М. Е. Семантика наречного слова в современном английском языке (на материале пространственных наречий) : дис. ... канд. филол. наук. Воронеж, 2015. 199 c.

5. Корнева В. В. Наречия и параметры пространственной картины мира. Воронеж : Воронежский государственный университет, 2008. 301 с.

6. Curme G. O. English Grammar. The Principles and Practice of English Grammar Applied to Present-Day Usage. New York : Barnes and Noble, 1966. 308 p.

7. Biber D., Conrad S., Leech G. Longman Student Grammar of Spoken and Written English. L. : Pearson Education Limited, 2003. 487 p.

8. Стернина М. A. Семантические типы наречного слова (на материале пространственных наречий английского и русского языков) : дис. ... канд. филол. наук. Воронеж, 1984. $188 \mathrm{c}$.

9. Кочетова Н. В. Национальная специфика семантем русской и английской адвербиальной лексики : дис. ... канд. филол. наук. Воронеж, 2017. 276 с.

10. Новый большой англо-русский словарь : в 3 т. / под ред. Ю. Д. Апресяна, Э. М. Медниковой. М. : Дрофа, 2003. $2496 \mathrm{c}$.

11. Cambridge Advanced Learner's Dictionary. URL: http://dictionary.cambridge.org/

12. Cobuild C. English Language Dictionary. Harper Collins Publishers, 1993. 1703 p.
13. Longman Dictionary of Contemporary English : with new words supplement. 3rd ed. Harlow: Longman, 2001. $1668 \mathrm{p}$.

14. The British National Corpus. URL: http://british-corpora.org

15. Кривенко Л. А. Национальная специфика семантем русской и английской субстантивной лексики : автореф. дис. ... канд. филол. наук. Воронеж, 2013. 22 с.

\section{REFERENCES}

1. Quirk R. et al. A Comprehensive Grammar of the English Language. L. : Longman, 1985. 1779 p.

2. Carter R., McCarthy M. Cambridge Grammar of English. A Comprehensive Guide to Spoken and Written English Grammar and Usage. Cambridge : Cambridge University Press, 2007. 973 p.

3. Khaimovich B. S., Rogovskaya B. I. Teoreticheskaya grammatika angliyskogo yazyka (na angliyskom yazyke) [Theoretical Grammar of the English Language (in English)]. Moscow : Vysshaya shkola, 1967. 298 p.

4. Pankratova M. E. Semantika narechnogo slova v sovremennom angliyskom yazyke (na materiale prostranstvennykh narechiy) [Semantics of Adverbs in Contemporary English (based on the Study of English Spatial Adverbs)]. $\mathrm{PhD}$ in Philological sci. diss. Voronezh : Voronezh State University, 2015. 199 p.

5. Korneva V. V. Narechiya I parametry prostranstvennoy kartiny mira [Adverbs and Parameters of Spatial Worldview] Voronezh : Voronezh State University, 2008. $301 \mathrm{p}$.

6. Curme G. O. English Grammar. The Principles and Practice of English Grammar Applied to Present-Day Usage. New York : Barnes and Noble, 1966. 308 p.

7. Biber D. Conrad S., Leech G. Longman Student Grammar of Spoken and Written English. L. : Pearson Education Limited, 2003. $487 \mathrm{p}$.

8. Sternina M. A. Semanticheskie tipy narechnogo slova (na materiale prostranstvennykh narechiy angliyskogo i russkogo yazykov) [Semantic types of English adverbs (Based on spatial English and Russian adverbs)]. PhD in Philological sci. diss. Voronezh : Voronezh State University, 1984. $188 \mathrm{p}$.

9. Kochetova N. V. Natsional'naya spetsifika semantem russkoy i angliyskoy adverbialnoy leksiki [National Specifics of the Semanthemes of Russian and English Adverbs]. $\mathrm{PhD}$ in Philological sci. diss. Voronezh : Voronezh State University, 2017. $276 \mathrm{p}$.

10. Novyi bolshoy anglo-russkiy slovar [New Big English-Russian Dictionary] / Ed. by Yu. D. Aprasyan, E. M. Mednikova. In 3 vol. Moscow: Drofa, 2003. 2496 p.

11. Cambridge Advanced Learner's Dictionary. Available at: http://dictionary.cambridge.org/

12. Cobuild C. English Language Dictionary. Harper Collins Publishers, 1993. 1703 p.

13. Longman Dictionary of Contemporary English: with new words supplement. 3rd ed. Harlow: Longman, 2001. $1668 \mathrm{p}$. 
14. The British National Corpus. Available at: http:// british-corpora.org

15. Krivenko L. A. Natsional'naya spetsifika semantem russkoy i angliyskoy substantivnoy leksiki [National

Воронежский институт Министерства внутренних дел Российской Федераџии

Панкратова М. Е., кандидат филологических наук, дочент кафедры иностранных языков

E-mail: marina.e.pankratova@gmail.com

Поступила в редакиию 1 декабря 2020 г.

Принята к публикачии 29 декабря 2020 г.

\section{Для цитирования:}

Панкратова М. Е. О статичной/динамичной вариантности семем английских пространственных наречий // Вестник Воронежского государственного университета. Серия: Лингвистика и межкультурная коммуникация. 2021. № 1. С. 83-88. DOI: https://doi.org/10.17308/ lic. $2021.1 / 3241$
Specifics of the Semanthemes of Russian and English Nouns]. Author's abstract of PhD in Philological sci. diss. Voronezh : Voronezh State University, 2013.

Voronezh Institute of the Ministry of Internal Affairs of the Russian Federation

Pankratova M. E., Candidate of Philology, Associate Professor of the Foreign Languages Department

E-mail: marina.e.pankratova@gmail.com

Received: 1 December 2020

Accepted: 29 Desember 2020

\section{For citation:}

Pankratova M. E. On static/dynamic variants of sememes of English spatial adverbs. Proceedings of Voronezh State University. Series: Linguistics and Intercultural Communication. 2021. No. 1. Pp. 83-88. DOI:https://doi. org/10.17308/lic.2021.1/3241 